\title{
Disks around hot stars in the Trifid nebula
}

\author{
B. Lefloch ${ }^{1}$, J. Cernicharo ${ }^{2}$, D. Cesarsky ${ }^{3}$, K. Demyk ${ }^{4}$, and L. F. Rodriguez ${ }^{5}$ \\ 1 Laboratoire d'Astrophysique de l'Observatoire de Grenoble, BP 53, 38041 Grenoble Cedex, France \\ 2 Consejo Superior de Investigaciones Científicas, Instituto de Estructura de la Materia, Serrano 123, \\ 28006 Madrid, Spain \\ 3 Max-Planck Institut für Extraterrestrische Physik, 85741 Garching, Germany \\ ${ }^{4}$ Institut d'Astrophysique Spatiale, Bât. 121, Université Paris XI, 91405 Orsay Cedex, France \\ 5 Instituto de Astronomía, UNAM, Campus Morelia, A.P. 3-72, Morelia, Mich. 58089, México
}

Received 8 December 2000 / Accepted 21 January 2001

\begin{abstract}
We report on mid-IR observations of the central region in the Trifid nebula, carried out with ISOCAM in several broad-band infrared filters and in the low resolution spectroscopic mode provided by the circular variable filter. Analysis of the emission indicates the presence of a hot dust component (500 to $1000 \mathrm{~K}$ ) and a warm dust component at lower temperatures $(\sim 150-200 \mathrm{~K})$ around several members of the cluster exciting the HII region, and other stars undetected at optical wavelengths. Complementary VLA observations suggest that the mid-IR emission could arise from a dust cocoon or a circumstellar disk, evaporated under the ionization of the central source and the exciting star of the nebula. In several sources the $9.7 \mu \mathrm{m}$ silicate band is seen in emission. One young stellar source shows indications of crystalline silicates in the circumstellar dust.
\end{abstract}

Key words. (ISM:) dust, extinction - (ISM:) HII regions - ISM: individual: Trifid - stars: formation

\section{Introduction}

The Trifid nebula is a nice example of a small HII region in an early stage of evolution. It is located at a distance of $1.68 \mathrm{kpc}$ (Lynds et al. 1985) and has an estimated dynamical age of 0.3-0.4 Myr (Lefloch \& Cernicharo 2000). Because of its youth and its small size, it provides us with a comprehensive picture of the early stages of massive starforming regions like Orion. Indeed, previous observations at millimeter wavelengths have shown that the protostellar cores surrounding the HII region have physical properties similar to the cores observed in Orion. These protostellar cores show good evidence that their birth was induced in the expansion of the HII region (Lefloch \& Cernicharo 2000).

The Trifid is excited by the star HD 164492A, classified as O7 V by Lynds et al. (1985). Five other stars (HD 164492B-F) were identified within $20^{\prime \prime}(0.16 \mathrm{pc})$ of HD 164492A, suggesting that the formation of a cluster accompanied the birth of the ionizing star. The spectral types determined for some of the sources indicate they are of intermediate mass (Kohoutek 1997). Here we report on mid-infrared ISOCAM observations of the central region in the HII region. These observations bring more insight of the star formation which accompanied the birth of the

Send offprint requests to: B. Lefloch, e-mail: lefloch@obs.ujf-grenoble.fr ionizing star. In particular, they reveal the lower mass star formation, embedded in the low-density ionized gas of the HII region. We find that many sources are surrounded by dense material, possibly in the form of disks photoevaporated by the ionizing radiation of the Trifid, similar to those found close to the Trapezium stars in Orion.

\section{Observations and results}

\subsection{The mid-infrared emission}

Several images of the Trifid were obtained with ISOCAM onboard ISO (Cesarsky et al. 1996) in the LW4 filter centered on the PAH band at $6.2 \mu \mathrm{m}(\Delta \lambda=1.0 \mu \mathrm{m})$ at $6^{\prime \prime}$ resolution, in the LW7 filter centered on the silicate band at $10 \mu \mathrm{m}(\Delta \lambda=3 \mu \mathrm{m})$ at $6^{\prime \prime}$ resolution and in the LW10 filter $(\lambda=11.5 \mu \mathrm{m}, \Delta \lambda=7 \mu \mathrm{m})$ at $3^{\prime \prime}$ resolution. The final maps are shown in Fig. 1. Several maps of the Trifid were obtained with the Circular Variable Filter (CVF) between 5 and $17 \mu \mathrm{m}$, with a spectral resolution $\lambda / \Delta \lambda \simeq 30$. Each map has a size of $32 \times 32$ pixels with an angular size of $3^{\prime \prime}$ per pixel.

Superposed to the extended emission from the dust lanes, we detect in the central LW4 image several pointlike sources (IRS 1 to IRS 5) near the stellar cluster (see Fig. 1d) and two more remote sources (IRS 6-7), apparent in Figs. 1c-e. The pointlike sources appear with the highest contrast in the LW4 filter; by comparison, IRS 4 
and IRS 5 are hardly visible in the LW7 and LW10 band. Except for the bright sources IRS 1-2 and IRS 6, the emission of the individual sources in the LW7 filter can hardly be separated from the extended emission of the dust lanes.

The maximum of emission (source IRS 1-2) peaks approximately $15^{\prime \prime}$ Southwest of HD $164492 \mathrm{~A}$ and coincides with the members of the central cluster HD 164492C-D. They are optically visible (Fig. 1a) and were classified as B6 V (Gahm et al. 1983) and Be LkHo123 (Herbig 1957) respectively. In the LW4 filter, the flux distribution is marginally resolved and indicates a size of $\sim 3^{\prime \prime}$ for the emitting region. The angular resolution of the infrared observations does not allow to distinguish the respective contributions of components C and D. However, a Gaussian fit to the CVF data gives a size of $2.5^{\prime \prime}$ for the emitting region. This size is very close to the projected distance between components C and D ( 4000 AU, Sect. 2.3) and suggests that both sources are contributing to the infrared flux; we identify IRS 1 (IRS 2) with component C (D) of the stellar cluster. All the other infrared sources are unresolved in our images.

\subsection{The physical properties of the dust}

The spectra of IRS 1-2, IRS 3 and IRS 6 between 5 and $17 \mu \mathrm{m}$ were obtained from the CVF data after subtracting the emission of a nearby $3 \times 3$ pixel $^{2}$ area used as a reference. The observed emission was fitted with a Gaussian function convolved with the Point Spread Function of the instrument. The IRS 1-2 spectrum is shown in Fig. 1f. It does not exhibit any narrow emission, apart from a weak NeII line at $12.7 \mu \mathrm{m}$. Remarkably, the silicate band at $9.7 \mu \mathrm{m}$ is detected in emission whereas it is seen in absorption in the dust lanes (not shown here).

We model the dust emission as a black-body law modified by a dust opacity $\tau_{\nu} \propto \nu$. The $5-17 \mu \mathrm{m}$ continuum emission is satisfactorily fitted by two components at temperatures $T \sim 500 \mathrm{~K}$ and $T \sim 200 \mathrm{~K}$ respectively (Fig. 1f). We adopted 0.2 " for the size of the "cold" component, as determined from the VLA observations (see below). The lack of constraints on the size of the hot component makes the amount of material difficult to quantify (especially the hot component). We derive typical hydrogen column densities $N(\mathrm{H}) \sim 10^{21} \mathrm{~cm}^{-2}$ for the component at $200 \mathrm{~K}$. The IRS 3 and IRS 6 spectra taken with the CVF display properties similar to IRS 1-2 (not shown). The continuum emission can be accounted for by the same simple model: one hot layer at $\sim 700-1000 \mathrm{~K}$ and a warm component at $\sim 150-300 \mathrm{~K}$.

The shape of the silicate band around $9.5 \mu \mathrm{m}$ (Fig. 1g) can help constrain the composition of the dust. The blue wing of the band $(8-10 \mu \mathrm{m})$ is well reproduced with amorphous pyroxene grains containing FeO inclusions (we assume spherical grains of $0.1 \mu \mathrm{m}$ size and the optical constants are taken from Henning et al. 1999). Comparison with laboratory transmission spectra of sub- micronic grains obtained at the IAS suggests that the broad shoulder longward of $11.5 \mu \mathrm{m}$ is probably due to crystalline silicates; it is well fitted with pure forsterite grains (Fig. 1g). We note that other crystalline silicates, such as pyroxenes, could also be present and account for the red shoulder. However, observations of other bands longward of $15 \mu \mathrm{m}$, like the $33.6 \mu \mathrm{m}$ forsterite band or the $40.5 \mu \mathrm{m}$ pyroxene band are necessary to confirm our identification and search for other disk components, like crystalline pyroxenes.

\subsection{The ionized material}

We observed the continuum radiation of the central region at $3.6 \mathrm{~cm}$ using the Very Large Array in its highest angular resolution configuration in 1998 March 13. The field was centered at $\alpha=18^{\mathrm{h}} 02^{\mathrm{m}} 27.223^{\mathrm{s}} \delta=-23^{\circ} 03^{\prime} 15.91^{\prime \prime}$ (Eq. 2000), so that all the infrared sources but IRS6 lie within the field. We used $1328+307$ as absolute amplitude calibrator and 1748-253 as the phase calibrator. A bootstrapped flux density of $0.271 \pm 0.001$ Jy was obtained for 1748-253. The observations were made in both circular polarizations with an effective bandwidth of $100 \mathrm{MHz}$. The rms noise in the map is $20 \mu \mathrm{Jy}_{\text {beam }}{ }^{-1}$; the beam size $(\mathrm{HPFW})$ is $0.38^{\prime \prime} \times 0.19^{\prime \prime}$ and the position angle is $11^{\circ}$. The map reveals the presence of two radio sources that coincide respectively with components $\mathrm{C}$ and $\mathrm{D}$ of the stellar cluster. We do not detect any emission from any other ISOCAM source, nor component B of the cluster, for which Yusef-Zadeh et al. (2000) report a $3.6 \mathrm{~cm}$ flux of $0.66 \mathrm{mJy}$, much higher than our $5-\sigma$ limit of $0.1 \mathrm{mJy}$. It appears unlikely that this emission arises from a circumstellar disk, as suggested by these authors; we propose that component B is a time variable gyrosynchrotron emitter, that may exhibit circular polarization in high-sensitivity radio-observations.

The emission from components $\mathrm{C}$ and $\mathrm{D}$ is compact and forms a close double, separated by $2.4^{\prime \prime}$. The flux densities at $3.6 \mathrm{~cm}$ were $1.64 \pm 0.02 \mathrm{mJy}$ (for C) and $1.48 \pm 0.02 \mathrm{mJy}$ (for D). Both sources appear unresolved with an angular size smaller than $\sim 0.2^{\prime \prime}$. No linear or circular polarization was found in the sources to an upper limit of $\sim 4 \%$ for the degree of polarization. The lack of polarization is consistent with a free-free interpretation for the observed emission. To obtain the spectral indices of both objets we undertook additional VLA observations at 2-cm in the C configuration during 1998 November 21. We used $0134+329$ as absolute amplitude calibrator and 1741-312 as the phase calibrator, with a bootstrapped flux density of $0.574 \pm 0.003 \mathrm{Jy}$. The flux densities at 2 -cm were $1.66 \pm 0.14 \mathrm{mJy}$ (for $\mathrm{C}$ ) and $1.30 \pm 0.14 \mathrm{mJy}$ (for $\mathrm{D})$, that combined with the 3.6-cm measurements imply spectral indices of $0.0 \pm 0.2$ and $-0.2 \pm 0.2$, for $\mathrm{C}$ and $\mathrm{D}$, respectively. These flat spectral indices in the centimeter range are characteristic of optically thin free-free emission. The measured fluxes are in rough agreement with similar observations at a lower angular resolution $\left(\sim 0.5^{\prime \prime}\right)$ by 
$\stackrel{\infty}{\circ}$

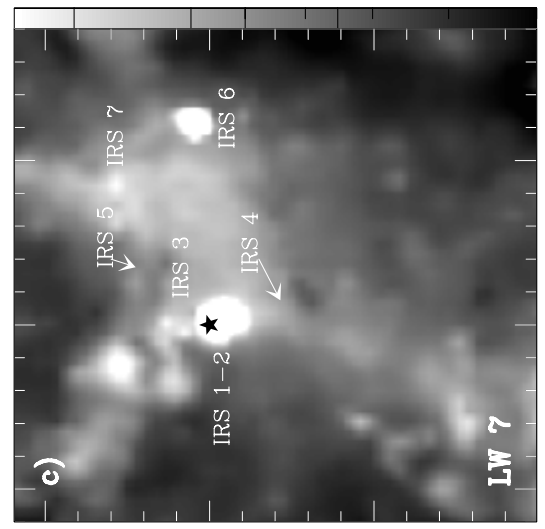

$\underset{-1}{8} \quad \stackrel{8}{-1}$

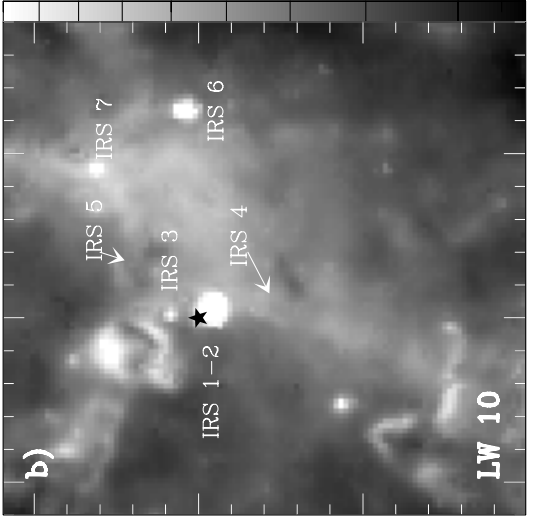

$\stackrel{8}{\circ}$

$\underset{1}{8}$

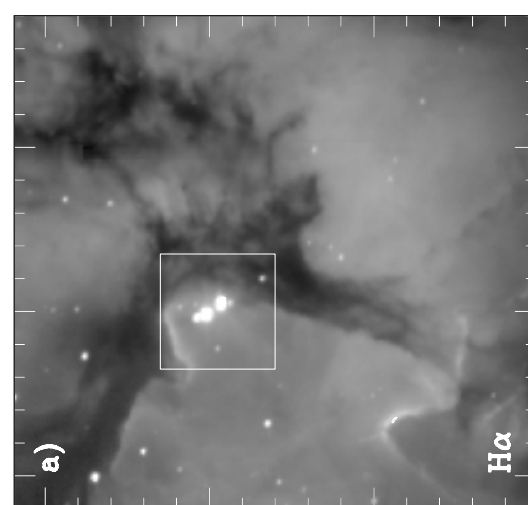

$\stackrel{\circ}{-}$

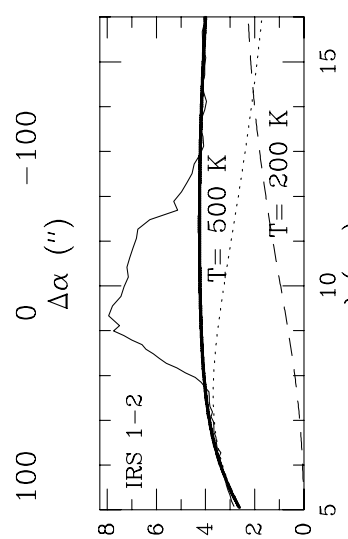

$=\left(\kappa_{\Gamma}\right)^{n}$

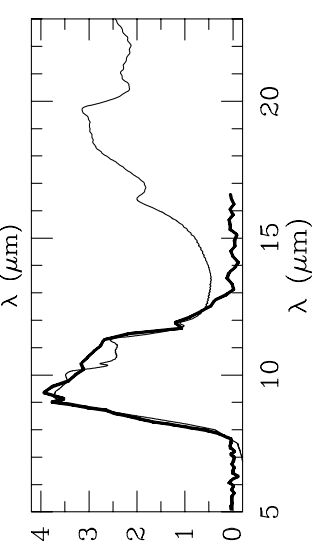

$\left(\kappa_{\Gamma}\right)^{\wedge}{ }^{A}$
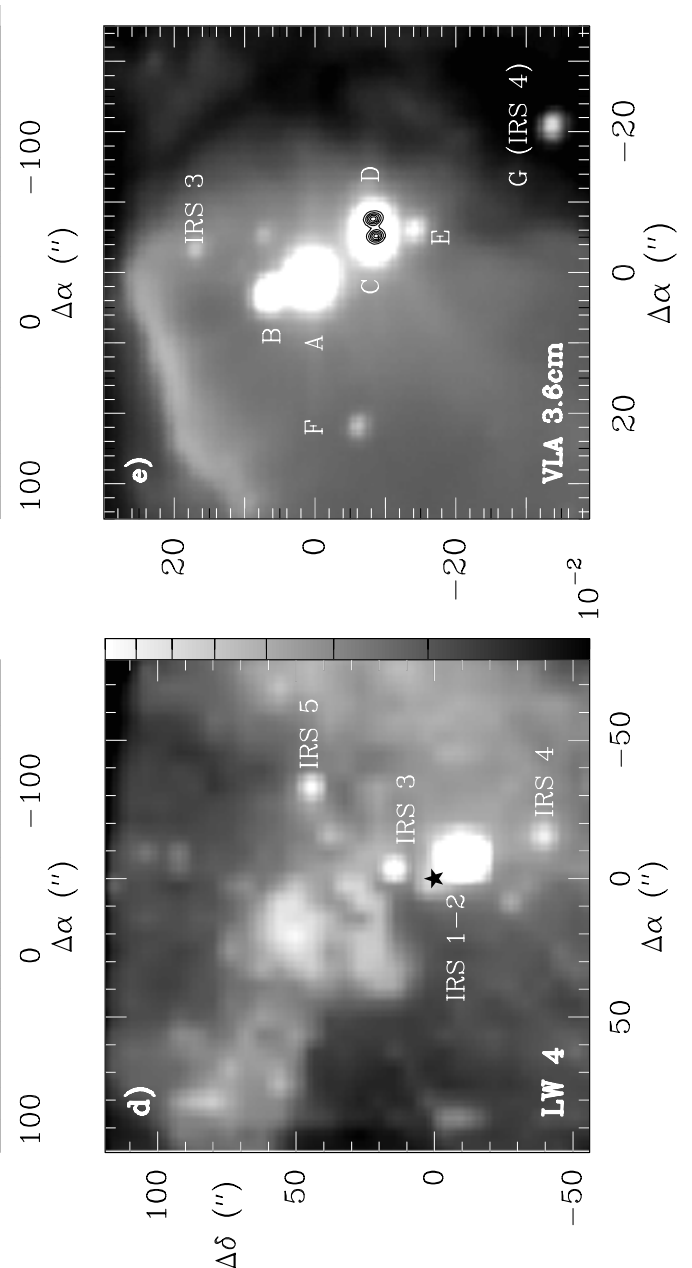

Fig. 1. a) Optical image of the $\mathrm{H} \alpha$ emission in the Trifid nebula taken with the IAC 80 telescope at the Observatorio del Teide, Tenerife, Spain (see also Cernicharo et al. 1998). Coordinates are in arcsec offsets with respect to the exciting star of the nebula HD 164492A : $\alpha_{2000}=18^{\mathrm{h}} 02^{\mathrm{m}} 23.55^{\mathrm{s}}, \delta_{2000}=-23^{\circ} 01^{\prime} 51.0^{\prime \prime}$ b) Infrared emission in the $8-15 \mu \mathrm{m}$ band (LW10). The black star marks the position of HD 164492A. c-d) Infrared emission in the 5.5-6.5 $\mu \mathrm{m}$ band (LW4) and 8.5-11.5 $\mu \mathrm{m}$ band (LW7) respectively. e) Magnified view of the $\mathrm{H} \alpha$ emission. We have superposed (contours) the $3.6 \mathrm{~cm}$ free-free emission observed at the VLA. The contours range from 0.2 to $1 \mathrm{mJy}$ by $0.2 \mathrm{mJy}_{\text {beam }}{ }^{-1}$. f) Spectral emission observed with the CVF towards IRS 1-2 and fit to the continuum. g) Silicate emission band observed towards IRS 1-2 after subtracting the continuum (thick) and fit to the emission (thin) assuming a mix of pure forsterite with amorphous pyroxenes with inclusions of $\mathrm{FeO}$ 
Yusef-Zadeh et al. (2000). From the observed emissivities and adopting a transverse size of $0.2^{\prime \prime}$ for the emitting region, we estimate a density of $\approx 10^{5} \mathrm{~cm}^{-3}$ and a total mass of $6.010^{-6} M_{\odot}$ for the ionized gas around $\mathrm{C}$ and $\mathrm{D}$.

\section{Discussion}

The color excesses measured by Kohoutek et al. (1997) towards the central stellar cluster $(\sim 0.3-0.4)$ indicate very low hydrogen column densities $N(\mathrm{H})=1.9-2.6 \times$ $20^{21} \mathrm{~cm}^{-2}$. Hence, there is no large-scale gas reservoir which could slow down the expansion of the ionized gas detected around components $\mathrm{C}$ and $\mathrm{D}$. The ionized gas must expand on the sonic timescale $\tau_{\mathrm{i}}=R_{\mathrm{i}} / c_{\mathrm{i}} \sim 80 \mathrm{yr}$, with $R_{\mathrm{i}}=0.1^{\prime \prime}$ as the radius of the region observed and $c_{\mathrm{i}}=10 \mathrm{~km} \mathrm{~s}^{-1}$. This timescale is so short compared to the age of the nebula that the free-free emission has to be sustained through the ionization of some "fresh" neutral material. The mid-infrared data provide direct evidence for some neutral material around components $\mathrm{C}$ and D. Therefore, we propose that the emission observed at the VLA and in the infrared originates from a reservoir of dense circumstellar material exposed to the ionizing radiation of HD $164492 \mathrm{~A}$, similar to the photo-evaporated disks detected in Orion (Churchwell et al. 1987; O'Dell et al. 1993).

Following the same approach as Churchwell et al. (1987), we estimate an ionizing flux $J_{\mathrm{i}}=1.610^{11} \mathrm{~cm}^{-2} \mathrm{~s}^{-1}$ at the ionization front and a mass-loss rate $\dot{M} \simeq$ $410^{-8} M_{\odot} \mathrm{yr}^{-1}$. It implies the mass of warm material detected in the infrared $\left(\sim 2-310^{-5} M_{\odot}\right)$ would have completely evaporated on a timescale of $400 \mathrm{yr}$, whereas the nebula is $\sim 0.3 \mathrm{Myr}$ old. The total circumstellar mass is probably much higher than the mass of material detected and the bulk of disk material corresponds to a colder component which emits at longer wavelengths. We also note that the mass of the photo-evaporated disks discovered in the Orion nebula are 100 times larger than the masses estimated here (Störzer \& Hollenbach 1999). The similarity between the mass of the ionized gas and the mid-infrared material suggests that we are detecting the emission of the photon-dominated region at the surface of the circumstellar disks. Interestingly, the column density of warm gas derived from the CVF data is in good agreement with the model of Johnstone et al. (1998) for the warm PDR gas at the surface of a photo-evaporated disk.

The only two infrared sources detected at the VLA are classified as massive or intermediate-mass objects; this suggests that all the infrared sources detected with ISOCAM have also low- or intermediate masses. This would naturally explain why neither IRS 3 nor IRS 4 were detected at the VLA, though at similar apparent distances to the ionizing source. However, we cannot exclude that we are misled by a projection effect and that the true distance to the exciting star is much larger. Apart from IRS 1-2, IRS 3 and IRS 6 exhibit the silicate band at $9.7 \mu \mathrm{m}$ in emission although both lay behind the Western dust lane of the nebula. It means that the dust tempera- ture of the circumstellar reservoir must be at least $\sim 200 \mathrm{~K}$. Since IRS 3 is very close to HD $164492 \mathrm{~A}$ and is also detected in the optical $\mathrm{H} \alpha$ line, one cannot exclude that the circumstellar dust is externally heated by the exciting star. Interestingly, this is not the case for other sources like IRS 5. This is probably because these sources are still embedded in their cocoon, and the outer heating is not sufficient to compete with the inner continuum source.

Only very few examples of crystalline silicates in protostellar disks have been reported so far (Malfait et al. $1998,1999)$. The mechanism responsible for the crystallization of silicates is unclear (Molster et al. 1999). Our observations in the Trifid nebula show that many young stellar sources are removing their parental cocoon, leaving their dense circumstellar disk exposed to the ionizing radiation of the exciting stars. In some sources we find a dust component at temperatures comparable to the glass temperature of silicates $\left(\sim 10^{3} \mathrm{~K}\right)$. We speculate that dust annealing in the PDR of the photoionized disks could contribute and maybe account for the crystalline component detected in IRS 1-2 (see e.g. Gail 1998). Complementary observations of IRS 1-2 with upcoming infrared instruments like SIRTF are necessary to confirm the presence of crystalline silicates, characterize their physical properties, and to constrain the possibility of such a scenario.

Acknowledgements. We acknowledge Spanish DGES for this supporting research under grants PB96-0883 and ESP981351E. LFR is grateful to CONACyT, México, for its support This research made use of SIMBAD.

\section{References}

Cernicharo, J., Lefloch, B., Cox, P., et al. 1998, Science, 282, 462

Cesarsky, C. J., Abergel, A., Agnese, P., et al. 1996, A\&A, 315, L32

Churchwell, E., Felli, M., Wood, D. O. S., \& Massi, M. 1987, ApJ, 321, 516

Gahm, G. F., Ahlin, P., \& Lindroos, K. P. 1983, A\&AS, 51, 143

Gail, H. P. 1998, A\&A, 332, 1099

Herbig, G. 1957, ApJ, 125, 654

Henning, T., Il'In, V. B., Krivova, N. A., et al. 1999, A\&A, 163,405

Johnstone, D., Hollenbach, D., \& Bally, J. 1998, ApJ, 499, 758

Kohoutek, L., Mayer, P., \& Lorenz, R. 1997, A\&AS, 134, 129

Lefloch, B., \& Cernicharo, J. 2000, ApJ, 545, 340

Lynds, B., Canzian, B. J., \& O’Neil, E. J. Jr. 1985, ApJ, 288, 164

Malfait, K., Waelkens, C., Waters, L. B. F. M., et al. 1998, A\&A, 332, L25

Malfait, K., Waelkens, C., Bouwman, J., De Koter, A., \& Waters, L. B. F. M. 1999, A\&A, 345, 181

Molster, F. J., Yamamura, I., Waters, L. B. F. M., et al. 1999, Nature, 401, 563

O’Dell, C. R., Wen, Z., \& Hu, X. 1993, ApJ, 410, 696

Störzer, H., \& Hollenbach, D. 1999, ApJ, 515, 669

Yusef-Zadeh, F., Shure, M., Wardle, M., \& Kassim, N. 2000, ApJ, 540, 842 